\title{
UNIVERSAL UNRAMIFIED COHOMOLOGY OF CUBIC FOURFOLDS CONTAINING A PLANE
}

\author{
ASHER AUEL, JEAN-LOUIS COLLIOT-THÉLÈNE, R. PARIMALA
}

\begin{abstract}
We prove the universal triviality of the third unramified cohomology group of a very general complex cubic fourfold containing a plane. The proof uses results on the unramified cohomology of quadrics due to Kahn, Rost, and Sujatha.
\end{abstract}

\section{INTRODUCTION}

Let $X$ be a smooth cubic fourfold, i.e., a smooth cubic hypersurface in $\mathbb{P}^{5}$. A well known problem in algebraic geometry concerns the rationality of $X$ over $\mathbb{C}$.

Expectation. The very general cubic fourfold over $\mathbb{C}$ is irrational.

Here, "very general" is usually taken to mean "in the complement of a countable union of proper Zariski closed subsets" in the moduli space of cubic fourfolds. At present, however, not a single cubic fourfold is provably irrational, though many families of rational cubic fourfolds are known.

If $X$ contains a plane $P$ (i.e., a linear two dimensional subvariety of $\mathbb{P}^{5}$ ), then $X$ is birational to the total space of a quadric surface bundle $\widetilde{X} \rightarrow \mathbb{P}^{2}$ by projecting from $P$. Its discriminant divisor $D \subset \mathbb{P}^{2}$ is a sextic curve. The rationality of a cubic fourfold containing a plane over $\mathbb{C}$ is also a well known problem.

Expectation. The very general cubic fourfold containing a plane over $\mathbb{C}$ is irrational.

Assuming that the discriminant divisor $D$ is smooth, the discriminant double cover $S \rightarrow \mathbb{P}^{2}$ branched along $D$ is then a K3 surface of degree 2 and the even Clifford algebra of the quadric fibration $\widetilde{X} \rightarrow \mathbb{P}^{2}$ gives rise to a Brauer class $\beta \in \operatorname{Br}(S)$, called the Clifford invariant of $X$. This invariant does not depend on the choice of $P$ (if $\beta$ is not zero, the plane $P$ is actually the unique plane contained in $X$ ). By classical results in the theory of quadratic forms (e.g., [40, Thm. 6.3]), $\beta$ is trivial if and only if the quadric surface bundle $\widetilde{X} \rightarrow \mathbb{P}^{2}$ has a rational section. Thus if $\beta$ is trivial then $X$ is rational (see [34, Thm. 3.1]), though it may happen that $X$ is rational even when $\beta$ is not trivial (see $[4$, Thm. 11]).

Some families of rational cubic fourfolds have been described by Fano [29], Tregub [51], [52], and Beauville-Donagi [8]. In particular, pfaffian cubic fourfolds, defined by pfaffians of skew-symmetric $6 \times 6$ matrices of linear forms, are rational. Hassett [33] describes, via lattice theory, Noether-Lefschetz divisors $\mathcal{C}_{d}$ in the moduli space $\mathcal{C}$ of cubic fourfolds. In particular, $\mathcal{C}_{14}$ is the closure of the locus of pfaffian cubic fourfolds and $\mathcal{C}_{8}$ is the locus of cubic fourfolds containing a plane. Hassett [34] identifies countably many divisors of $\mathcal{C}_{8}$ - the union of which is Zariski dense in $\mathcal{C}_{8}$ - consisting of rational cubic fourfolds with trivial Clifford invariant. 
A natural class of birational invariants arise from unramified cohomology groups. The unramified cohomology of a rational variety is trivial (i.e., reduces to the cohomology of the ground field), cf. [18, Thm. 1.5] and [17, §2 and Thm. 4.1.5]. Such invariants have been known to provide useful obstructions to rationality.

For a smooth cubic fourfold $X$ over $\mathbb{C}$, the unramified cohomology groups

$$
H_{\mathrm{nr}}^{i}(X / \mathbb{C}, \mathbb{Q} / \mathbb{Z}(i-1))
$$

vanish for $0 \leq i \leq 3$, see Theorem 2.4. The case $i=1$ follows from the Kummer sequence (see Theorem 2.4). For $i=2$, one appeals to the Leray spectral sequence and a version of the Lefschetz hyperplane theorem due to M. Noether, as in [45, Thm. A.1]. For $i=3$, the proof relies on the integral Hodge conjecture for cycles of codimension 2 on smooth cubic fourfolds, a result proved by Voisin [56, Thm. 18] building on [44] and [58].

Something stronger is known when $i \leq 2$, namely that for any field extension $F / \mathbb{C}$, the natural map $H^{i}(F, \mathbb{Q} / \mathbb{Z}(i-1)) \rightarrow H_{\mathrm{nr}}^{i}\left(X_{F} / F, \mathbb{Q} / \mathbb{Z}(i-1)\right)$ is an isomorphism, see Theorem 2.4. In this case, we say that the unramified cohomology is universally trivial. The universal behavior of an unramified cohomology group can lead to a finer obstruction to rationality. While we do not know if the unramified cohomology of an arbitrary smooth cubic fourfold over $\mathbb{C}$ is universally trivial in degree 3 , our main result is the following.

Theorem 1. Let $X \subset \mathbb{P}^{5}$ be a very general cubic fourfold containing a plane over $\mathbb{C}$. Then $H_{\mathrm{nr}}^{3}(X / \mathbb{C}, \mathbb{Q} / \mathbb{Z}(2))$ is universally trivial, i.e., the natural map $H^{3}(F, \mathbb{Q} / \mathbb{Z}(2)) \rightarrow$ $H_{\mathrm{nr}}^{3}\left(X_{F} / F, \mathbb{Q} / \mathbb{Z}(2)\right)$ is an isomorphism for every field extension $F / \mathbb{C}$.

Here, "very general" means that the quadric surface bundle $\widetilde{X} \rightarrow \mathbb{P}^{2}$ attached to the plane $P \subset X$ has simple degeneration (see Proposition 4.1) and that the associated K3 surface $r: S \rightarrow \mathbb{P}^{2}$ of degree 2 satisfies $r^{*}: \operatorname{Pic}\left(\mathbb{P}^{2}\right) \rightarrow \operatorname{Pic}(S)$ is an isomorphism. A very general cubic fourfold containing a plane has nontrivial Clifford invariant. Theorem 1 implies the corresponding statement with $\mu_{2}^{\otimes 2}$ coefficients as well (see Corollary 5.6).

This article is organized as follows. In $\S 1$, we expand on the notion of universal triviality and, more generally, universal torsion by a positive integer, of the Chow group $A_{0}(X)$ of zero-cycles of degree zero on a smooth proper variety $X$. We state an extension of a theorem of Merkurjev [43, Thm. 2.11] on the relationship between universal torsion properties of $A_{0}(X)$ and analogous properties for unramified cohomology groups and, more generally, unramified classes in cycle modules. We also recall that rationally connected varieties, and among them Fano varieties, satisfy such a universal torsion property for $A_{0}(X)$.

In $\S 2$, we discuss the specific case of cubic hypersurfaces $X \subset \mathbb{P}^{n+1}$ for $n \geq 2$. Already in this case, the universal triviality is an open question. We register a folklore proof that $A_{0}(X)$ is killed by 18 , and by 2 as soon as $X$ contains a $k$-line. In particular, for any smooth cubic hypersurface $X \subset \mathbb{P}^{n+1}$ over $\mathbb{C}$, with $n \geq 2$, and any field extension $F / \mathbb{C}$, the group $A_{0}\left(X_{F}\right)$ is 2-torsion, which also follows from the existence of a unirational parameterization of $X$ of degree 2 . The only possible interesting unramified cohomology groups are thus those with coefficients $\mathbb{Z} / 2 \mathbb{Z}$. We recall the known results on these groups. Our main result, Theorem 1, then appears as one step beyond what was known, but still leaving open the question of the universal triviality of $A_{0}(X)$. 
In $\S 3$, we recall some results on the unramified cohomology groups of quadrics in degrees at most 3. The most important is due to Kahn, Rost, and Sujatha (see Theorem 3.3), who build upon earlier work of Merkurjev, Suslin, and Rost.

In $\S 4$, we discuss the fibration into 2-dimensional quadrics over the projective plane, as well as its corresponding even Clifford algebra, associated to a smooth cubic fourfold containing a given plane.

In $\S 5$, we use the results of the previous two sections to prove Theorem 1 . The "very general" hypothesis allows us to construct well-behaved parameters at the local rings of curves on $\mathbb{P}^{2}$ that split in $S$ (see Lemma 5.3).

We thank the American Institute of Mathematics for sponsoring the workshop "Brauer groups and obstruction problems: moduli spaces and arithmetic" held February 25 to March 1, 2013, in Palo Alto, California. This work emerges from a problem session group formed during the workshop, and involved the participation of Marcello Bernardara, Jodi Black, Evangelia Gazaki, Andrew Kresch, Eric Riedl, Olivier Wittenberg, and Matthew Woolf. We thank Andrew Kresch for further discussions after the AIM meeting.

The first author is partially supported by National Science Foundation grant MSPRF DMS-0903039 and by an NSA Young Investigator grant. The third author is partially supported by National Science Foundation grant DMS-1001872.

\section{UnRAMified COHOMOLOGY AND CHOW GROUP OF 0-CYClES}

1.1. Unramified elements. A general framework for the notion of "unramified element" is established in $[17, \S 2]$. Let $k$ be a field and denote by Local $_{k}$ the category of local $k$-algebras together with local $k$-algebra homomorphisms. Let $\mathrm{Ab}$ be the category of abelian groups and let $M:$ Local $_{k} \rightarrow$ Ab be a functor. For any field $K / k$ the group of unramified elements of $M$ in $K / k$ is the intersection

$$
M_{\mathrm{nr}}(K / k)=\bigcap_{k \subset \mathscr{O} \subset K} \operatorname{im}(M(\mathscr{O}) \rightarrow M(K))
$$

over all rank 1 discrete valuations rings $k \subset \mathscr{O} \subset K$ with $\operatorname{Frac}(\mathscr{O})=K$.

There is a natural map $M(k) \rightarrow M_{\mathrm{nr}}(K / k)$ and we say that the group of unramified elements $M_{\mathrm{nr}}(K / k)$ is trivial if this map is surjective.

For $X$ an integral scheme of finite type over a field $k$, in this paper we write $M_{\mathrm{nr}}(X / k):=M_{\mathrm{nr}}(k(X) / k)$. By this definition, the group $M_{\mathrm{nr}}(X / k)$ is a $k$-birational invariant of integral schemes of finite type over $k$. We will be mostly concerned with the functor $M=H_{\text {ét }}^{i}(-, \mu)$ with coefficients $\mu$ either $\mu_{2}^{\otimes(i-1)}$ (under the assumption $\operatorname{char}(k) \neq 2$ ) or

$$
\mathbb{Q} / \mathbb{Z}(i-1):=\lim _{\longrightarrow} \mu_{m}^{\otimes(i-1)},
$$

the limit being taken over all integers $m$ coprime to the characteristic of $k$. In this case, $M_{\mathrm{nr}}(X / k)$ is called the unramified cohomology group $H_{\mathrm{nr}}^{i}(X, \mu)$ of $X$ with coefficients in $\mu$.

Theorem 1.1. Let $k$ be a field of characteristic different from a prime $p$. The natural map

$$
H^{i}\left(F, \mu_{p}^{\otimes(i-1)}\right) \rightarrow H^{i}(F, \mathbb{Q} / \mathbb{Z}(i-1))
$$

is injective. 
This is a well known consequence of the norm residue isomorphism theorem (previously known as the Bloch-Kato conjecture) in degree $i-1$. We use this only for $i=3$, in which case it is a consequence of the Merkurjev-Suslin theorem. For $i=3, p=2$, this is a theorem of Merkurjev.

If $F / k$ is a field extension, we write $X_{F}=X \times_{k} F$. If $X$ is geometrically integral over $k$, we say that $M_{\mathrm{nr}}(X / k)$ is universally trivial if $M_{\mathrm{nr}}\left(X_{F} / F\right)$ is trivial for every field extension $F / k$.

Proposition 1.2 ([17, $\S 2$ and Thm. 4.1.5]). Let $M:$ Local $_{k} \rightarrow$ Ab be a functor satisfying the following conditions:

- If $\mathscr{O}$ is a discrete valuation ring containing $k$, with fraction field $K$ and residue field $\kappa$, then $\operatorname{ker}(M(\mathscr{O}) \rightarrow M(K)) \subset \operatorname{ker}(M(\mathscr{O}) \rightarrow M(\kappa))$.

- If $A$ is a regular local ring of dimension 2 containing $k$, with fraction field $K$, then $\operatorname{im}(M(A) \rightarrow M(K))=\bigcap_{\mathrm{ht}(\mathfrak{p})=1} \operatorname{im}\left(M\left(A_{\mathfrak{p}}\right) \rightarrow M(K)\right)$.

- The group $M_{\mathrm{nr}}\left(\mathbb{A}_{k}^{1} / k\right)$ is universally trivial.

Then $M_{\mathrm{nr}}\left(\mathbb{P}_{k}^{n} / k\right)$ is universally trivial.

The functor $H_{\text {ét }}^{i}(-, \mu)$ satisfies the conditions of Proposition 1.2 (cf. [17, Thm. 4.1.5]), hence if $X$ is a $k$-rational variety, then $H_{\mathrm{nr}}^{i}(X / k, \mu)$ is universally trivial.

Denote by $A b^{\bullet}$ the category of graded abelian groups. An important class of functors $M$ : Local $_{k} \rightarrow \mathrm{Ab}^{\bullet}$ arise from the cycle-modules of Rost [48, Rem. 5.2]. In particular, unramified cohomology arises from a cycle-module. A cycle module $M$ comes equipped with residue maps of graded degree -1

$$
M^{i}(k(X)) \stackrel{\partial}{\rightarrow} \bigoplus_{x \in X^{(1)}} M^{i-1}(k(x))
$$

for any integral $k$-variety $X$. If $X$ is smooth and proper, then the kernel is $M_{\mathrm{nr}}^{i}(X / k)$.

1.2. Chow groups of $\mathbf{0}$-cycles. Denote by $\mathrm{CH}_{d}(X)$ the Chow group of $d$-cycles on a smooth variety $X$ over a field $k$ up to rational equivalence. If $X$ is proper over $k$, then there is a well-defined degree map $\mathrm{CH}_{0}(X) \rightarrow \mathbb{Z}$, and we denote by $A_{0}(X)$ its kernel, called the Chow group of 0 -cycles of degree 0. The group $A_{0}(X)$ is a $k$-birational invariant of smooth, proper, integral varieties over a field $k$, see [21, Prop. 6.3] requiring resolution of singularities and [30, Ex. 16.1.11] in general. The computation of the Chow groups of projective space goes back to Severi. For 0-cycles, one easily sees $A_{0}\left(\mathbb{P}_{k}^{n}\right)=0$.

For $X$ a proper $k$-variety, we say that $A_{0}(X)$ is universally trivial if $A_{0}\left(X_{F}\right)=0$ for every field extension $F / k$. To check triviality of $A_{0}\left(X_{F}\right)$ over every field extension $F / k$ seems like quite a burden. However, usually it suffices to check it over the function field by the following well known lemma.

Lemma 1.3. Let $X$ be a geometrically irreducible smooth proper variety over a field $k$. Assume that $X$ has a 0-cycle of degree 1. The group $A_{0}(X)$ is universally trivial if and only if $A_{0}\left(X_{k(X)}\right)=0$.

Proof. If $A_{0}(X)$ is universally trivial then $A_{0}\left(X_{k(X)}\right)=0$ by definition. Let us prove the converse. Write $d=\operatorname{dim}(X)$. Let $\xi \in X_{k(X)}$ be the $k(X)$-rational point which is the image of the "diagonal morphism" Spec $k(X) \rightarrow X \times{ }_{k} \operatorname{Spec} k(X)$. Let $P$ be a fixed 0 -cycle of degree 1 on $X$. By hypothesis, we have $\xi=P_{k(X)}$ in $\mathrm{CH}_{0}\left(X_{k(X)}\right)$. The closures of $P_{k(X)}$ and $\xi$ in $X \times_{k} X$ are $P \times_{k} X$ and the diagonal 
$\Delta_{X}$, respectively. By the closure in $X \times_{k} X$ of a 0-cycle on $X_{k(X)}$, we mean the sum, taken with multiplicity, of the closures of each closed point in the support of the 0 cycle on $X_{k(X)}$. Hence the class of $\Delta_{X}-P \times_{k} X$ is in the kernel of the contravariant map $\mathrm{CH}^{d}\left(X \times_{k} X\right) \rightarrow \mathrm{CH}^{d}\left(X_{k(X)}\right)$. Since $\mathrm{CH}^{d}\left(X_{k(X)}\right)$ is the inductive limit of $\mathrm{CH}^{d}\left(X \times_{k} U\right)$ over all dense open subvarieties $U$ of $X$, we have that $\Delta_{X}-P \times_{k} X$ vanishes in some $\mathrm{CH}^{d}\left(X \times{ }_{k} U\right)$. We thus have a decomposition of the diagonal

$$
\Delta_{X}=P \times_{k} X+Z
$$

in $\mathrm{CH}^{d}\left(X \times_{k} X\right)$, where $Z$ is a cycle with support in $X \times V$ for some proper closed subvariety $V \subset X$.

Now, each $d$-cycle $T$ on $X \times{ }_{k} X$ induces a homomorphism $T_{*}: \mathrm{CH}_{0}(X) \rightarrow \mathrm{CH}_{0}(X)$ defined by $T_{*}(z)=\left(p_{1}\right)_{*}\left(T \cdot p_{2}^{*} z\right)$, where $p_{i}: X \times_{k} X \rightarrow X$ are the two projections. The map $T \mapsto T_{*}$ is itself a homomorphism

$$
\mathrm{CH}^{d}\left(X \times_{k} X\right)=\mathrm{CH}_{d}\left(X \times_{k} X\right) \rightarrow \operatorname{Hom}_{\mathbb{Z}}\left(\mathrm{CH}_{0}(X), \mathrm{CH}_{0}(X)\right)
$$

by [30, Cor. 16.1.2]. We note that $\left(\Delta_{X}\right)_{*}$ is the identity map and $\left(P \times_{k} X\right)_{*}(z)=$ $\operatorname{deg}(z) P$. By the easy moving lemma for 0 -cycles on a smooth variety (cf. [19, p. 599]), for a proper closed subvariety $V \subset X$, every 0-cycle on $X$ is rationally equivalent to one with support away from $V$. This implies that $Z_{*}=0$ for any $d$-cycle with support on $X \times_{k} V$ for a proper closed subvariety $V \subset X$. Thus if $A_{0}\left(X_{k(X)}\right)=0$, then by the decomposition of the diagonal (1), we see that the identity map restricted to $A_{0}(X)$ is zero.

For any field extension $F / k$, we have the base-change $\Delta_{X_{F}}=P_{F} \times_{F} X_{F}+Z_{F}$ of the decomposition of the diagonal (1), hence the same argument as above shows that $A_{0}\left(X_{F}\right)=0$. We conclude that $A_{0}(X)$ is universally trivial.

Let $M$ be a cycle module and let $X$ be a smooth, proper, geometrically connected variety over the field $k$. Let $N$ be a positive integer. We say that $M_{\mathrm{nr}}(X / k)$ is universally $N$-torsion if the cokernel of the natural map $M(F) \rightarrow M_{\mathrm{nr}}\left(X_{F} / F\right)$ is killed by $N$ for every field extension $F / k$. We say that $A_{0}(X)$ is universally $N$ torsion if $A_{0}\left(X_{F}\right)$ is killed by $N$ for every field extension $F / k$.

The index $i(X)$ of a variety $X$ is the smallest positive degree of a 0 -cycle.

Theorem 1.4. Let $X$ be a smooth proper geometrically connected variety over a field $k$. Let $N>0$ be an integer. If the Chow group $A_{0}(X)$ of 0 -cycles of degree 0 is universally $N$-torsion then for every cycle module $M$ over $k$, the group $M_{\mathrm{nr}}(X / k)$ is universally $i(X) N$-torsion.

Proof. This is a direct consequence of Lemma 1.3 and [37, Prop. RC.8].

The case $N=1$ is a generalization of a theorem of Merkurjev [43, Thm. 2.11], who also establishes a converse statement. For any $N>0$, one may extend Merkurjev's method to prove a weak converse: if $M_{\mathrm{nr}}(X / k)$ is universally $N$-torsion for every cycle module $M$ then $A_{0}(X)$ is also universally $N$-torsion.

We point out that the appearance of the index of $X$, in the statement of Theorem 1.4, is necessary. For any quadric $X$ over any field $k$ of characteristic $\neq 2$, we have that $A_{0}(X)$ is universally trivial (see [50]). However, if $X$ is the 4-dimensional quadric associated to an anisotropic Albert form $X$ over a field $k$ of characteristic $\neq 2$, then $\operatorname{coker}\left(H^{3}(F, \mathbb{Q} / \mathbb{Z}(2)) \rightarrow H_{\mathrm{nr}}^{3}(X / k, \mathbb{Q} / \mathbb{Z}(2))\right) \cong \mathbb{Z} / 2 \mathbb{Z}$ by [36, Thm. 5]. 
Hence $A_{0}(X)$ is universally trivial, while there are nontrivial unramified elements of some cycle module over $X$. Note that the index of an anisotropic quadric is 2 .

The following lemmas will be used in the next section.

Lemma 1.5. Let $X$ be a proper variety over a field $k$ with $X(k) \neq \emptyset$. Let $N>0$ be an integer. If for every finite extension $K / k$ and any two $K$-points $P, Q \in X(K)$ the class of the 0 -cycle $P-Q$ is $N$-torsion in $A_{0}\left(X_{K}\right)$, then $A_{0}(X)$ is $N$-torsion.

Proof. Fixing $P \in X(k)$, any element of $A_{0}(X)$ can be written as a linear combination of 0 -cycles of degree 0 of the form $Z-\operatorname{deg}(Z) P$, where $Z$ is a closed point of $X$. Let $K$ be the residue field of $Z$. Consider the morphism $f: X_{K} \rightarrow X$. Since $K \otimes_{k} K$ has $K$ as a direct factor, there is a corresponding $K$-rational point $\zeta$ of $X_{K}$ lying over $Z$, such that $f_{*} \zeta=P$. By hypothesis $\zeta-P_{K}$ is $N$-torsion, hence $f_{*}\left(\zeta-P_{K}\right)=Z-\operatorname{deg}(Z) P$ is $N$-torsion.

Lemma 1.6. Let $k$ be an algebraically closed field and $K / k$ a field extension. Let $X$ be a smooth projective connected variety over $k$. Then the natural map $\mathrm{CH}_{0}(X) \rightarrow$ $\mathrm{CH}_{0}\left(X_{K}\right)$ is injective.

Proof. Let $z$ be a 0 -cycle on $X$ that becomes rationally equivalent to zero on $X_{K}$. Then there exists a subextension $L$ of $K / k$ that is finitely generated over $k$, such that $z$ becomes rationally equivalent to zero on $X_{L}$. In fact, we can spread out the rational equivalence to a finitely generated $k$-algebra $A$. Since $k$ is algebraically closed, there are many $k$-points on $\operatorname{Spec} A$, at which we can specialize the rational equivalence.

Lemma 1.7. Let $X$ be a smooth proper connected variety over an algebraically closed field $k$ of infinite transcendence degree over its prime field. If $A_{0}(X)=0$ then there exists an integer $N>0$ such that $A_{0}(X)$ is universally $N$-torsion.

Proof. The variety $X$ is defined over an algebraically closed subfield $L \subset k$, with $L$ algebraic over a field finitely generated over its prime field. That is, there exists a variety $X_{0}$ over $L$ with $X \cong X_{0} \times_{L} k$. Let $\eta$ be the generic point of $X_{0}$. Let $P$ be an $L$-point of $X_{0}$. One may embed the function field $F=L\left(X_{0}\right)$ into $k$, by the transcendence degree hypothesis on $k$. Let $K$ be the algebraic closure of $F$ inside $k$. By Lemma 1.6 and the hypothesis that $A_{0}(X)=0$, we have that $A_{0}\left(X_{0} \times_{L} F\right)=0$. This implies that there is a finite extension $E / F$ of fields such that $\eta_{E}-P_{E}=0$ in $A_{0}\left(X_{0} \otimes_{L} E\right)$. Taking the corestriction to $F$, one finds that $N\left(\eta_{F}-P_{F}\right)=0$ in $A_{0}\left(X_{0} \times{ }_{L} F\right)$, hence in $A_{0}(X)$ as well. Arguing as in the proof of Lemma 1.3, we conclude that $A_{0}(X)$ is universally $N$-torsion.

1.3. Connections with complex geometry. The universal torsion of $A_{0}(X)$ puts strong restrictions on the variety $X$. For example, the following result is well known.

Proposition 1.8. Let $X$ be a smooth proper geometrically irreducible variety over a field $k$ of characteristic zero. If $A_{0}(X)$ is universally $N$-torsion for some positive integer $N$ then, $H^{0}\left(X, \Omega_{X}^{i}\right)=0$ and $H^{i}\left(X, \mathscr{O}_{X}\right)=0$ for all $i \geq 1$.

Proof. Over a complex surface, the result goes back to Bloch [9, App. Lec. 1], by exploiting a decomposition of the diagonal and the action of cycles on various cohomology theories. Aspects of this argument were developed in [12]. A proof over 
the complex numbers can be found in [55, Cor. 10.18, $\S 10.2 .2]$. Over a general field of characteristic zero, the argument is sketched in [28, p. 187].

Over $\mathbb{C}$, the universal triviality of $A_{0}(X)$ does not imply $H^{0}\left(X, \omega_{X}^{\otimes n}\right)=0$ for all $n>1$. Otherwise, a surface over $\mathbb{C}$ with $A_{0}(X)=0$ would additionally satisfy $P_{2}(X)=h^{0}\left(X, \omega_{X}^{\otimes 2}\right)=0$, hence would be rational by Castelnovo's criterion.

It is however well known that there exist nonrational complex surfaces $X$, with $p_{g}(X)=q(X)=0$ and for which $A_{0}(X)=0$. Enriques surfaces were the first examples, extensively studied in [26], [27, p. 294] with some examples considered earlier in [47], see also [15]. For more examples, see [10]. We remark that for an Enriques surface $X$, we have that $H_{\mathrm{nr}}^{1}(X / \mathbb{C}, \mathbb{Z} / 2 \mathbb{Z})=H_{\text {ét }}^{1}(X, \mathbb{Z} / 2 \mathbb{Z})=\mathbb{Z} / 2 \mathbb{Z}$. Hence by Theorem 1.4, we see that $A_{0}(X)$ is not universally trivial.

The following result was stated without detailed proof as the last remark of [12]. As we show, it is an immediate consequence of a result in [22]. A more geometric proof was recently shown to us by C. Voisin.

Proposition 1.9. Let $X$ be a smooth proper connected surface over $\mathbb{C}$. Suppose that all groups $H_{\mathrm{Betti}}^{i}(X(\mathbb{C}), \mathbb{Z})$ are torsionfree and that $A_{0}(X)=0$. Then $A_{0}(X)$ is universally trivial.

Proof. By Lemma 1.7, we have that $A_{0}(X)$ is universally $N$-torsion. Hence by Lemma 1.8, we have that $H^{i}\left(X, \mathscr{O}_{X}\right)=0$ for all $i \geq 1$. Hence $p_{g}(X)=q(X)=0$, and thus $b_{3}(X)=b_{1}(X)=2 q(X)=0$. The torsion-free hypothesis on cohomology finally allows us conclude, from $[22$, Thm. $3.10(\mathrm{~d})]$, that $A_{0}\left(X_{F}\right)=0$ for any field extension $F / \mathbb{C}$.

Corollary 1.10. Let $X$ be a smooth proper connected surface over $\mathbb{C}$. Suppose that the Néron-Severi group $\mathrm{NS}(X)$ of $X$ is torsionfree and that $A_{0}(X)=0$. Then $A_{0}(X)$ is universally trivial.

Proof. The group $H_{\text {Betti }}^{1}(X(\mathbb{C}), \mathbb{Z})$ is clearly torsionfree. For any smooth proper connected variety $X$ over $\mathbb{C}$ of dimension $d$ there is an isomorphism $N S(X)_{\text {tors }} \simeq$ $H_{\text {Betti }}^{2}(X(\mathbb{C}), \mathbb{Z})_{\text {tors }}$, and the finite groups $H_{\text {Betti }}^{2}(X(\mathbb{C}), \mathbb{Z})_{\text {tors }}$ and $H_{\text {Betti }}^{2 d-1}(X(\mathbb{C}), \mathbb{Z})_{\text {tors }}$ are dual to each other. The hypotheses thus imply that for the surface $X$, all groups $H_{\text {Betti }}^{i}(X(\mathbb{C}), \mathbb{Z})$ are torsionfree, and we apply Proposition 1.9.

The first surfaces $S$ of general type with $p_{g}(S)=q(S)=0$ were constructed in [14] and [31]. Simply connected surfaces $X$ of general type for which $p_{g}(X)=0$ were constructed by Barlow [6], who also proved that $A_{0}(X)=0$ for some of them. See also the recent paper [53]. For such surfaces, $\operatorname{Pic}(X)=\operatorname{NS}(X)$ has no torsion, thus Corollary 1.10 applies. The group $A_{0}(X)$ is universally trivial, but the surfaces are far from being rational, since they are of general type.

A smooth projective variety $X$ over a field $k$ is called rationally chain connected if for every algebraically closed field extension $K / k$, any two $K$-points of $X$ can be connected by a chain of rational curves. Smooth, geometrically unirational varieties are rationally connected. It is a theorem of Campana [13] and Kollár-MiyaokaMori [41] that any smooth projective Fano variety is rationally chain connected. If $X$ is rationally chain connected, then $A_{0}\left(X_{K}\right)=0$ for any algebraically closed field extension $K / k$. While a standard argument then proves that $A_{0}\left(X_{F}\right)$ is torsion for 
every field extension $F / k$, the following more precise result, in the spirit of Lemma 1.7 above, is known.

Proposition 1.11 ([19, Prop. 11]). Let $X$ be a smooth, projective, rationally chain connected variety over a field $k$. Then there exists an integer $N>0$ such that $A_{0}(X)$ is universally $N$-torsion.

There exist rationally connected varieties $X$ over an algebraically closed field of characteristic zero with $A_{0}(X)$ not universally trivial. Indeed, let $X$ be a unirational threefold with $H_{\mathrm{nr}}^{2}(X, \mathbb{Q} / \mathbb{Z}(1)) \cong \operatorname{Br}(X) \neq 0$, see e.g., [2]. Then by Theorem 1.4, $A_{0}(X)$ is not universally trivial.

We do not know whether there exist smooth Fano varieties over an algebraically closed field of characteristic zero with $A_{0}(X)$ not universally trivial.

\section{Chow groups of 0-CyCles on Cubic hypersurfaces}

Now we will discuss the situation for cubic hypersurfaces $X \subset \mathbb{P}^{n+1}$ with $n \geq 2$. Quite a few years ago, one of us learned the following argument from the Dean of Trinity College, Cambridge.

Proposition 2.1. Let $X \subset \mathbb{P}_{k}^{n+1}$, with $n \geq 2$, be an arbitrary cubic hypersurface over an arbitrary field $k$. Then $A_{0}(X)$ is 18-torsion. If $X(k) \neq \emptyset$ then $A_{0}(X)$ is 6-torsion. If $X$ contains a k-line, then $A_{0}(X)$ is 2-torsion.

Proof. Assuming the assertion in the case $X(k) \neq \emptyset$, we can deduce the general case from a norm argument, noting that $X$ acquires a rational point after an extension of degree 3. So we assume that $X(k) \neq \emptyset$. By Lemma 1.5, to prove the general statement over an arbitrary field $k$, it suffices to prove that for any distinct points $P, Q \in X(k)$, the class of the 0 -cycle $P-Q$ is 6 -torsion, and that it is 2 -torsion if $X$ contains a $k$-line. Choose a $\mathbb{P}_{k}^{3} \subset \mathbb{P}_{k}^{n+1}$ containing $P$ and $Q$. If $X$ contains a $k$-line $L$, take such a $\mathbb{P}_{k}^{3}$ which contains the line $L$. Then $X \cap \mathbb{P}_{k}^{3}$ is either $\mathbb{P}_{k}^{3}$ or is a cubic surface in $\mathbb{P}_{k}^{3}$. In the first case, $P$ is rationally equivalent to $Q$ on $\mathbb{P}_{k}^{3}$, hence on $X$. Thus we can assume, for the rest of the proof, that $X \subset \mathbb{P}_{k}^{3}$ is a cubic surface, possibly singular. We will sketch a route to prove the result, leaving the minute details to the reader.

We first show that if the cubic surface $X$ contains a $k$-line $L$, then for any two distinct points $P, Q \in X(k)$, we have $2(P-Q)=0 \in A_{0}(X)$. It is enough to prove this when $Q$ is a $k$-point on $L$. If $P$ also lies on $L$, then $P-Q=0$. If $P$ does not lie on $L$, let $\Pi$ be the plane spanned by $P$ and $L$. If it is contained in $X$, then clearly $P-Q=0$. If not, then it cuts out on $X$ a cubic curve $C$, one component of which is the line $L$, the other component is a conic. At this juncture, we leave it to the reader to consider the various possible cases and show that $2(P-Q)$ is rationally equivalent to zero on $C$, hence on $X$. The coefficient 2 is the degree of intersection of the conic with the line.

We may now assume that the cubic surface $X$ does not contain a $k$-line. If $P$ and $Q$ were both singular, then the line through $P$ and $Q$ would intersect the surface with multiplicity at least 4 , hence would be contained in $X$, which is excluded. We may thus assume that $P$ is a regular $k$-point. First assume that $Q$ is singular. Let $\Pi$ be a plane which contains $P$ and $Q$. It is not contained in $X$. Its trace on $X$ is a cubic curve $C$ in the plane $\Pi$, such that $C$ is singular at $Q$. A discussion of cases 
then shows that $6(Q-P)=0$ on $C$, hence on $X$ (the occurence of 6 rather than 2 , comes from allowing nonperfect fields in characteristic 3). Now suppose that both $P$ and $Q$ are regular, hence smooth, $k$-points. Let $T_{P} \subset \mathbb{P}_{k}^{3}$ be the tangent plane to $X$ at $P$, and $T_{Q} \subset \mathbb{P}_{k}^{3}$ the tangent plane to $X$ at $Q$. Since $X$ contains no $k$-line, the tangent planes are distinct. Let $L=T_{P} \cap T_{Q}$ be their intersection. This line is not contained in $X$, which it intersects in a zero-cycle $z$ of degree 3 over $k$. The trace of $X$ on the plane $T_{P}$ is a cubic curve $C_{P}$ which is singular at $P$ and contains the 0 -cycle $z$. We leave it to the reader to check that $6 P-2 z=0 \in A_{0}\left(C_{P}\right)$, hence in $A_{0}(X)$. Similarly, $6 Q-2 z=0 \in A_{0}\left(C_{Q}\right)$, hence in $A_{0}(X)$. Thus $6(P-Q)=0 \in A_{0}(X)$ in all cases.

For smooth cubic hypersurfaces, the last part of Proposition 2.1 is a consequence of the fact that a cubic hypersurface containing a line has unirational parameterizations of degree 2. This fact that was likely known to M. Noether (cf. [16, App. B]).

Theorem 2.2. Let $X \subset \mathbb{P}_{k}^{n+1}$, with $n \geq 2$, be a smooth cubic hypersurface containing a $k$-line $L$. Then $X$ has a unirational parameterization of degree 2.

Proof. Denote by $W$ the variety of pairs $(p, l)$ where $p \in L$ and $l$ is a line in $\mathbb{P}_{k}^{n+1}$ tangent to $X$ at $p$. Then the projection $W \rightarrow L$ is a Zariski locally trivial $\mathbb{P}^{n-1}$ bundle. A general such line $l$ intersects $X$ in one further point, defining a rational map $g: W \rightarrow X$. This map is two-to-one. Indeed, as before, for a general point $p \in X$, the plane through $p$ and $L$ meets $X$ in the union of $L$ and a smooth conic. Generally, that conic meets $L$ in two points. The lines through $p$, and tangent to $X$, are exactly those connecting $p$ to these two points of intersection.

We can give another argument, following [32, §2.1]. Projecting from $L$ displays $X$ as birational to the total space of a conic bundle $Y \rightarrow \mathbb{P}_{k}^{n-1}$, where $Y$ is the blow-up of $X$ in $L$. Each point $P$ in the base $\mathbb{P}_{k}^{n-1}$ corresponds to a plane containing $L$, and intersecting $X$ with this plane is the union of $L$ and a conic $C_{P}$; this conic is the fiber above $P$ in the conic bundle. Let $M$ be the incidence variety of pairs $(P, p)$ where $P \in \mathbb{P}_{k}^{n-1}$ and $p \in L$, such that $p \in C_{P}$. Then the projection $M \rightarrow \mathbb{P}_{k}^{n-1}$ has degree 2 , the fiber over each $P$ is exactly the two points of intersection of $L$ and $C_{P}$. The other projection $M \rightarrow L$ displays $M$ as the total space of a Zariski locally trivial $\mathbb{P}^{n-2}$-bundle, hence $M$ is rational. Then consider the base change of $Y \rightarrow \mathbb{P}_{k}^{n-1}$ by $M \rightarrow \mathbb{P}_{k}^{n-1}$. The resulting conic bundle $M \times_{\mathbb{P}_{k}^{n-1}} Y \rightarrow M$ has a tautological rational section. Thus $M \times_{\mathbb{P}_{k}^{n-1}} Y$ is rational (being a conic bundle over $M$ with a rational section) and the projection to $Y$ has degree 2.

Over an algebraically closed field, any cubic hypersurface $X \subset \mathbb{P}^{n+1}$, with $n \geq 2$, contains a line. Indeed, by taking hyperplane sections, one is reduced to the well knwon fact that any cubic surface $X \subset \mathbb{P}^{3}$ contains a line over an algebraically closed field, see [46, Prop. 7.2] for instance. Proposition 2.1 and Theorem 1.4 then yield the following corollary.

Corollary 2.3. Let $X \subset \mathbb{P}_{k}^{n+1}$, with $n \geq 2$, be a smooth cubic hypersurface over a field $k$. If $X$ contains a $k$-line (e.g., if $k$ is an algebraically closed field) then $A_{0}(X)$ is universally 2-torsion and thus for every cycle module $M$ over $k$, the group $M_{\mathrm{nr}}(X / k)$ is universally 2-torsion.

Hassett and Tschinkel $[35, \S 7.5]$ prove that the cubic fourfolds over $\mathbb{C}$ with a unirational parameterization of odd degree are Zariski dense in the moduli space. 
In particular, they prove that on a Zariski dense subset of the Noether-Lefschetz divisor $\mathcal{C}_{d}$, with $d=2\left(m^{2}+m+1\right)$ for $m \geq 1$, the cubic fourfolds have a unirational parameterization of degree $m^{2}+m+1$. The countable union of these loci is dense in the moduli space. Together with Corollary 2.3, this implies that such cubic fourfolds $X$ have universally trivial $A_{0}(X)$, and thus for every cycle module $M$ over $\mathbb{C}$, the group $M_{\mathrm{nr}}(X / k)$ is universally trivial.

Corollary 2.3 leaves the following questions open. Let $X \subset \mathbb{P}^{n+1}$, with $n \geq 2$, be a smooth cubic hypersurface over $\mathbb{C}$.

a) Is the group $A_{0}(X)$ universally trivial?

b) For any integer $i \geq 1$, are the unramified cohomology groups $H_{\mathrm{nr}}^{i}\left(X / \mathbb{C}, \mu_{2}^{\otimes(i-1)}\right)$ universally trivial?

The following theorem gathers previously known results.

Theorem 2.4. Let $X \subset \mathbb{P}_{k}^{n+1}$ be a smooth cubic hypersurface over a field $k$ of characteristic zero. Then $H_{\mathrm{nr}}^{i}(X / k, \mathbb{Q} / \mathbb{Z}(1))$ is universally trivial for all $n \geq 3$ and $0 \leq i \leq 2$. If $k=\mathbb{C}$, then $H_{\mathrm{nr}}^{3}(X / k, \mathbb{Q} / \mathbb{Z}(2))$ is trivial for all $3 \leq n \leq 4$.

Proof. Let $F / k$ be any field extension. For any complete intersection $Y \subset \mathbb{P}_{F}^{n+1}$ of dimension $\geq 3$ over $F$, the restriction map on Picard groups $\operatorname{Pic}\left(\mathbb{P}_{F}^{n+1}\right) \rightarrow \operatorname{Pic}(Y)$ is an isomorphism and the natural map on Brauer groups $\operatorname{Br}(F) \rightarrow \operatorname{Br}(Y)$ is an isomorphism, see [45, Thm. A.1]. By purity, for any smooth variety $Y$ over $F$, we have that $H_{\mathrm{nr}}^{1}(Y / F, \mathbb{Q} / \mathbb{Z}(1))=H_{\text {ét }}^{1}(Y, \mathbb{Q} / \mathbb{Z}(1))$ and that $H_{\mathrm{nr}}^{2}(Y / F, \mathbb{Q} / \mathbb{Z}(1))=\operatorname{Br}(Y)$, see [23, Cor. 3.2, Prop. 4.1] and [11]. From the Kummer sequence for a projective and geometrically connected variety $X$ over $F$, we get an the exact sequence

$$
1 \rightarrow F^{\times} / F^{\times n} \rightarrow H_{\text {ét }}^{1}\left(X, \mu_{n}\right) \rightarrow \operatorname{Pic}(X)[n] \rightarrow 0
$$

which yields an exact sequence

$$
0 \rightarrow H^{1}(F, \mathbb{Q} / \mathbb{Z}(1)) \rightarrow H_{\text {ét }}^{1}(X, \mathbb{Q} / \mathbb{Z}(1)) \rightarrow \operatorname{Pic}(X)_{\text {tors }} \rightarrow 0 .
$$

upon taking direct limits.

When $X \subset \mathbb{P}_{k}^{n+1}$ is a smooth cubic hypersurface, the above considerations imply that $H_{\mathrm{nr}}^{i}(X / k, \mathbb{Q} / \mathbb{Z}(1))$ is universally trivial for $i=1,2$. Also $H_{\mathrm{nr}}^{0}(X / k, \mathbb{Q} / \mathbb{Z}(1))$ is universally trivial since $X$ is geometrically irreducible.

Now assume $k=\mathbb{C}$. If $n=3$, then $X$ contains a line hence is birational to a conic fibration over $\mathbb{P}^{2}$, as in the proof of Theorem 2.2. For a conic bundle $Y$ over a complex surface, one has $H_{\mathrm{nr}}^{3}(Y / \mathbb{C}, \mathbb{Q} / \mathbb{Z}(2))=0$ (cf. [20, Cor. 3.1(a)]). If $n=4$ and $X$ contains a plane, then $X$ is birational to a fibration $Y \rightarrow \mathbb{P}^{2}$ in 2-dimensional quadrics, and once again $[20$, Cor. $3.1(\mathrm{a})]$ yields $H_{\mathrm{nr}}^{3}(X / \mathbb{C}, \mathbb{Q} / \mathbb{Z}(2))=0$.

For $n \geq 2$ arbitrary, $X$ is unirational hence rationally chain connected. Then $[25$, Thm. 1.1] implies that the integral Hodge conjecture for codimension 2 cycles on $X$ is equivalent to the vanishing of $H_{\mathrm{nr}}^{3}(X / \mathbb{C}, \mathbb{Q} / \mathbb{Z}(2))$.

For smooth cubic threefolds, the integral Hodge conjecture holds for codimension 2 cycles, as $H^{4}(X, \mathbb{Z})$ is generated by a line. This yields another proof of $H_{\mathrm{nr}}^{3}(X / \mathbb{C}, \mathbb{Q} / \mathbb{Z}(2))=0$ in the case $n=3$.

For smooth cubic fourfolds, the integral Hodge conjecture for codimension $2 \mathrm{cy}-$ cles is a result of Voisin [56, Thm. 18], building on [44] and [58]. We thus get $H_{\mathrm{nr}}^{3}(X / \mathbb{C}, \mathbb{Q} / \mathbb{Z}(2))=0$ for an arbitrary smooth cubic hypersurface $X \subset \mathbb{P}^{5}$.

Our main result, Theorem 1, is a first step beyond the mentioned results. 


\section{UNRAMIFIED COHOMOLOGY OF QUADRICS}

Let $Q$ be a smooth quadric over a field $k$ of characteristic $\neq 2$ defined by the vanishing of a quadratic form $q$. We note that the dimension of $Q$ (as a $k$-variety) is 2 less than the dimension of $q$ (as a quadratic form). When $Q$ has even dimension, one defines the discriminant $d(Q) \in H^{1}\left(k, \mu_{2}\right)$ of $Q$ to be the (signed) discriminant of $q$. If $Q$ has even dimension and trivial discriminant or has odd dimension, then define the Clifford invariant $c(Q) \in \operatorname{Br}(k)$ of $Q$ to be the Clifford invariant of $q$, i.e., the Brauer class of the Clifford algebra $C(q)$ or the even Clifford algebra $C_{0}(q)$, respectively. We point out when $q$ has even rank and trivial discriminant, then a choice of splitting of the center induces a decomposition $C_{0}(q) \cong C_{0}^{+}(q) \times C_{0}^{-}(q)$, with $C(q), C_{0}^{+}(q)$, and $C_{0}^{-}(q)$ all Brauer equivalent central simple $k$-algebras. Under the given constraints on dimension and discriminant, these invariants only depend on the similarity class of $q$, and thus yield well-defined invariants of $Q$.

The following two results are well known (cf. [1, §5, p. 485]; see also the proof of [24, Théorème 2.5]), though we could not find the second stated in the literature.

Theorem 3.1. Let $k$ be a field of characteristic $\neq 2$. Let $Q$ be a smooth quadric surface over $k$. Then

$$
\operatorname{ker}(\operatorname{Br}(k) \rightarrow \operatorname{Br}(k(Q)))= \begin{cases}0 & \text { if } d(Q) \text { is nontrivial } \\ \mathbb{Z} / 2 \mathbb{Z} \cdot c(Q) & \text { if } d(Q) \text { is trivial }\end{cases}
$$

where $c(Q)$ is the Clifford invariant of $Q$.

Proposition 3.2. Let $k$ be a field of characteristic $\neq 2$. Let $Q$ be a quadric surface cone over $k$, the base of which is a smooth conic $Q_{0}$. Then

$$
\operatorname{ker}(\operatorname{Br}(k) \rightarrow \operatorname{Br}(k(Q)))=\mathbb{Z} / 2 \mathbb{Z} \cdot c\left(Q_{0}\right)
$$

where $c\left(Q_{0}\right)$ is the Clifford invariant of $Q_{0}$.

Proof. In this case $k(Q) \cong k\left(Q_{0} \times_{k} \mathbb{P}_{k}^{1}\right)$, hence $\operatorname{ker}(\operatorname{Br}(k) \rightarrow \operatorname{Br}(k(Q)))$ equals $\operatorname{ker}\left(\operatorname{Br}(k) \rightarrow \operatorname{Br}\left(k\left(Q_{0}\right)\right)\right)$. Thus the proposition follows from the case of smooth conics, a result going back to Witt [57, Satz p. 465].

Finally, the deepest result we will need is the following one concerning the degree three unramified cohomology of a quadric.

Theorem 3.3 (Kahn-Rost-Sujatha [36, Thm. 5]). Let $k$ be a field of characteristic $\neq 2$. Let $Q$ be a smooth quadric surface over $k$. Then the natural map

$$
H^{3}(k, \mathbb{Q} / \mathbb{Z}(2)) \rightarrow H_{\mathrm{nr}}^{3}(Q / k, \mathbb{Q} / \mathbb{Z}(2))
$$

is surjective.

The following is an amplification of one the main results of Arason's thesis.

Theorem 3.4. Let $k$ be a field of characteristic $\neq 2$. Let $Q$ be a smooth quadric surface over $k$ defined by a nondegenerate quadratic form $q$ of rank 4 . Then the kernel of the map $H^{3}(k, \mathbb{Q} / \mathbb{Z}(2)) \rightarrow H^{3}(k(Q), \mathbb{Q} / \mathbb{Z}(2))$ coincides with the kernel of the map $H^{3}\left(k, \mu_{2}^{\otimes 2}\right) \rightarrow H^{3}\left(k(Q), \mu_{2}^{\otimes 2}\right)$, and it is equal to the set of symbols

$$
\{(a, b, c): q \text { is similar to a subform of } \ll-a,-b,-c \gg\} \text {. }
$$


Proof. By a standard norm argument, the kernel of $H^{3}(k, \mathbb{Q} / \mathbb{Z}(2)) \rightarrow H^{3}(k(Q), \mathbb{Q} / \mathbb{Z}(2))$ is 2-torsion. By Merkurjev's theorem (see Theorem 1.1), the two kernels in the Theorem thus coincide. The precise description of the kernel with coefficients $\mathbb{Z} / 2 \mathbb{Z}$ is Arason's [1, Satz 5.6].

However, for our purposes, we will only need to know that certain special symbols are contained in this kernel. We can give a direct proof of this fact.

Lemma 3.5. Let $k$ be a field of characteristic $\neq 2$. If $\langle 1,-a,-b, a b d\rangle$ is isotropic over $k$, then for $w$ any norm from $k(\sqrt{d}) / k$, the symbol $(a, b, w) \in H^{3}\left(k, \mu_{2}^{\otimes 2}\right)$ is trivial.

Proof. Put $l=k(\sqrt{d})$. As $\langle 1,-a,-b, a b d\rangle$ is isotropic, there exist $x, y, u, v \in k$ such that $x^{2}-a y^{2}=b\left(u^{2}-a d v^{2}\right) \neq 0$. The class $\left(a, x^{2}-a y^{2}\right)$ is trivial, hence

$$
(a, b, w)=\left(a,\left(x^{2}-a y^{2}\right) /\left(u^{2}-a d v^{2}\right), w\right)=\left(a, u^{2}-a d v^{2}, w\right) .
$$

Let $w=N_{l / k}\left(w^{\prime}\right)$. By the projection formula, we have that

$$
(a, b, w)=\operatorname{cores}_{k(\sqrt{d}) / k}\left(a, u^{2}-a d v^{2}, w^{\prime}\right)
$$

which is trivial since $\left(a, u^{2}-a d v^{2}\right)=\left(a, u^{2}-a(\sqrt{d} v)^{2}\right) \in H^{2}\left(l, \mu_{2}^{\otimes 2}\right)$ is trivial.

\section{Cubic fourfolds containing a Plane and Clifford algebras}

Let $X$ be a smooth cubic fourfold over a field $k$. Suppose $X \subset \mathbb{P}_{k}^{5}=\mathbb{P}(V)$ contains a plane $P=\mathbb{P}(W)$, where $W \subset V$ is a dimension 3 linear subspace of $V$. Let $\widetilde{X}$ be the blow-up of $X$ along $P$ and $\pi: \widetilde{X} \rightarrow \mathbb{P}(V / W)$ the projection from $P$. We will write $\mathbb{P}_{k}^{2}=\mathbb{P}(V / W)$. Then the blow-up of $\mathbb{P}_{k}^{5}$ along $P$ is isomorphic to the total space of the projective bundle $p: \mathbb{P}(\mathscr{E}) \rightarrow \mathbb{P}_{k}^{2}$, where $\mathscr{E}=\left(W \otimes \mathscr{O}_{\mathbb{P}_{k}^{2}}\right) \oplus \mathscr{O}_{\mathbb{P}_{k}^{2}}(-1)$, and in which $\pi: \widetilde{X} \rightarrow \mathbb{P}_{k}^{2}$ embeds as a quadric surface bundle.

Now choose homogeneous coordinates $\left(x_{0}: x_{1}: x_{2}: y_{0}: y_{1}: y_{2}\right)$ on $\mathbb{P}_{k}^{5}$. Since $\operatorname{Aut}_{k}\left(\mathbb{P}^{5}\right)$ acts transitively on the set of planes in $\mathbb{P}_{k}^{5}$, without loss of generality, we can assume that $P=\left\{x_{0}=x_{1}=x_{2}=0\right\}$. Write the equation of $X$ as

$$
\sum_{0 \leq m \leq n \leq 2} a_{m n} y_{m} y_{n}+\sum_{0 \leq p \leq 2} b_{p} y_{p}+c=0
$$

for homogeneous linear polynomials $a_{m n}$, quadratic polynomials $b_{p}$, and a cubic polynomial $c$ in $k\left[x_{0}, x_{1}, x_{2}\right]$. Then we define a quadratic form $q: \mathscr{E} \rightarrow \mathscr{O}_{\mathbb{P}_{k}^{2}}(1)$ over $\mathbb{P}_{k}^{2}$ by

$$
q\left(y_{0}, y_{1}, y_{2}, z\right)=\sum_{0 \leq m \leq n \leq 2} a_{m n} y_{m} y_{n}+\sum_{0 \leq p \leq 2} b_{p} y_{p} z+c z^{2}
$$

on local sections $y_{i}$ of $\mathscr{O}_{\mathbb{P}_{k}^{2}}$ and $z$ of $\mathscr{O}_{\mathbb{P}_{k}^{2}}(-1)$. Of course, given $X$, the quadratic form $q$ is only well-defined up to multiplication by an element of $\Gamma\left(X, \mathbb{G}_{\mathrm{m}}\right)=k^{\times}$. The quadric fibration associated to $\left(\mathscr{E}, q, \mathscr{O}_{\mathbb{P}_{k}^{2}}(1)\right)$ is precisely $\pi: \widetilde{X} \rightarrow \mathbb{P}_{k}^{2}$. The associated bilinear form $b_{q}: \mathscr{E} \rightarrow \mathscr{E} \vee \otimes \mathscr{O}_{\mathbb{P}_{k}^{2}}(1)$ has Gram matrix

$$
\left(\begin{array}{cccc}
2 a_{00} & a_{01} & a_{02} & b_{0} \\
a_{01} & 2 a_{11} & a_{12} & b_{1} \\
a_{02} & a_{12} & 2 a_{22} & b_{2} \\
b_{0} & b_{1} & b_{2} & 2 c
\end{array}\right)
$$


whose determinant $\Delta$ is a homogeneous sextic polynomial defining the discriminant divisor $D \subset \mathbb{P}_{k}^{2}$.

Proposition 4.1. Let $X$ be a smooth cubic fourfold containing a plane $P$ over a field $k$ of characteristic $\neq 2$. Denote by $\pi: \widetilde{X} \rightarrow \mathbb{P}_{k}^{2}$ the associated quadric surface bundle, $D \subset \mathbb{P}_{k}^{2}$ the discriminant divisor, and $U=\mathbb{P}_{k}^{2} \backslash D$. Then the following are equivalent:

a) The divisor $D$ is smooth over $k$.

b) The fibers of $q$ are nondegenerate over points of $U$ and have a radical of dimension 1 over points of $D$.

c) The fibers of $\pi$ are smooth quadric surfaces over points of $U$ and are quadric surface cones with isolated singularity over points of $D$.

d) There is no other plane in $X \times_{k} \bar{k}$ meeting $P \times_{k} \bar{k}$.

In this case, we say that $\pi$ has simple degeneration.

Proof. The equivalence between $a$ and $b$ is proved in [7, I Prop. 1.2(iii)] over an algebraically closed field and [3, Prop. 1.6] in general. The equivalence of $b$ and $c$ follows from the fact that the singular locus of a quadric is the projectivization of its radical. The statement that $d$ implies $a$ appears without proof in [54, $\$ 1$ Lemme 2], and holds over a general field. Finally, another plane intersecting $P$ nontrivially will give rise, in the projection, to a singular line or plane in a fiber of the quadric fibration, contradicting $c$.

Let $\mathscr{C}_{0}$ be the even Clifford algebra associated to $\left(\mathscr{E}, q, \mathscr{O}_{\mathbb{P}_{k}^{2}}(1)\right)$, cf. [3, §1.5]. It is a locally free $\mathscr{O}_{\mathbb{P}_{k}^{2}}$-algebra of rank 8 whose center $\mathscr{Z}$ is a locally free quadratic

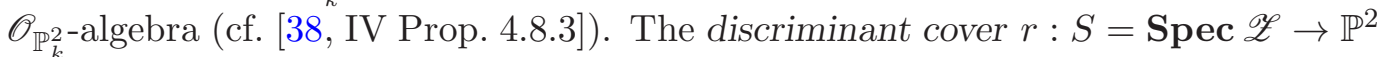
is a finite flat double cover branched along the sextic $D \subset \mathbb{P}_{k}^{2}$.

Assuming simple degeneration, then $S$ is a smooth K3 surface of degree 2 over $k$. We say that a cubic fourfold $X$ containing a given plane $P$ is very general if the quadric surface bundle associated to $P$ has simple degeneration and the associated K3 surface $S$ satisfies $r^{*}: \operatorname{Pic}\left(\mathbb{P}_{k}^{2}\right) \rightarrow \operatorname{Pic}(S)$ is an isomorphism. If the ground field $k$ is algebraically closed, then for any overfield $F$ of $k$, by rigidity of the Néron-Severi group, this implies that $r^{*}: \operatorname{Pic}\left(\mathbb{P}_{F}^{2}\right) \rightarrow \operatorname{Pic}\left(S_{F}\right)$ is an isomorphism. We note that in the moduli space $\mathcal{C}_{8}$ of smooth cubic fourfolds containing a plane over $\mathbb{C}$, the locus of very general ones (in our definition) is the complement of countably many proper Zariski closed subvarieties (see [33, Thm. 1.0.1]). Thus this notion of very general agrees with the usual notion in algebraic geometry.

A cubic fourfold $X$ containing a plane $P$ over $\mathbb{C}$ is very general if and only if the Chow group $\mathrm{CH}^{2}(X)$ of cycles of codimension 2 is spanned by the classes of $P$ and of a fiber $Q$ of the quadric fibration (see [54, $\S 1$ Prop. 2] and its proof). A very general cubic fourfold $X$ has nontrivial Clifford invariant. Indeed, the Zariski closure of a rational section of the quadric fibration would provide a cycle of dimension 2 having one point of intersection with a general fiber $Q$. Such a cycle cannot be rationally equivalent to any linear combination of $P$ and $Q$, since both $P$ and $Q$ have even intersection with $Q$. Hence $X$ cannot be very general (see [34, Thm. 3.1]).

Still assuming simple degeneration, the even Clifford algebra $\mathscr{C}_{0}$, considered over its center, defines an Azumaya quaternion algebra $\mathscr{B}_{0}$ over $S$ (cf. [42, Prop. 3.13] 
and [5, Prop. 1.13]). We refer to the Brauer class $\beta_{X, P} \in \operatorname{Br}(S)$ of $\mathscr{B}_{0}$ as the Clifford invariant of the pair $(X, P)$.

Lemma 4.2. Let $X$ be a smooth cubic fourfold containing a plane $P$ over a field $k$ of characteristic $\neq 2$. Assume that the quadric surface bundle associated to $P$ has simple degeneration. Let $S$ be the associated K3 surface of degree 2. If $X$ contains another plane $P^{\prime}$ (necessarily skew to $P$ ), then the fibration $\pi: \widetilde{X} \rightarrow \mathbb{P}_{k}^{2}$ has a rational section. In this case, the generic fiber of $\pi$ is an isotropic quadric over $k\left(\mathbb{P}^{2}\right)$, hence is a $k\left(\mathbb{P}^{2}\right)$-rational variety. In particular, the $k$-variety $X$ is $k$-rational. Moreover, $\beta_{X, P}=0 \in \operatorname{Br}(S)$.

Proof. Recall how the fibration $\pi: \widetilde{X} \rightarrow \mathbb{P}_{k}^{2}$ is constructed. One fixes an arbitrary plane $Q \subset \mathbb{P}_{k}^{5}$ which does not meet $P$. The morphism $\pi$ is induced by the morphism $\varpi: X \backslash P \rightarrow Q$ sending a point $x$ of $X$ not on $P$ to the unique point of intersection of the linear space spanned by $P$ and $x$ with the linear space $Q$.

Let $P^{\prime}$ be another plane in $X$. By Proposition 4.1, $P^{\prime}$ must be skew to $P$. Take the plane $Q$ to be $P^{\prime}$. On points of $P^{\prime} \subset X$ the map $\varpi: X \backslash P^{\prime} \rightarrow P^{\prime}$ is the identity. Thus $\pi$ has a rational section, the generic fiber is an isotropic quadric, hence rational over $k\left(\mathbb{P}^{2}\right)$, and the even Clifford invariant of this quadric is trivial.

In view of this lemma, when given a smooth cubic fourfold containing a plane $P$ whose associated quadric fibration has simple degeneration, we shall abuse notation and write $\beta_{X} \in \operatorname{Br}(S)$ instead of $\beta_{X, P} \in \operatorname{Br}(S)$.

The generic fiber of $\left(\mathscr{E}, q, \mathscr{O}_{\mathbb{P}_{k}^{2}}(1)\right)$ is a quadratic form of rank 4 over $k\left(\mathbb{P}^{2}\right)$ with values in a $k\left(\mathbb{P}^{2}\right)$-vector space of dimension 1 . Choosing a generator $l$ of $\mathscr{O}_{\mathbb{P}_{k}^{2}}(1)$ over $k\left(\mathbb{P}^{2}\right)$, we arrive at a usual quadratic form $(E, q)$ with discriminant extension $k(S) / k\left(\mathbb{P}^{2}\right)$. The generic fiber of $\beta_{X}$ is then in the image of the restriction map $\operatorname{Br}\left(k\left(\mathbb{P}^{2}\right)\right) \rightarrow \operatorname{Br}(k(S))$ by the fundamental relations for the even Clifford algebra (cf. [39, Thm. 9.12]). Explicitly, the full Clifford algebra $C(E, q)$ is central simple over $k\left(\mathbb{P}^{2}\right)$ and its restriction to $k(S)$ is Brauer equivalent to the even Clifford algebra $C_{0}(E, q)$, hence with the generic fiber of $\beta_{X}$. We note that $C(E, q)$ depends on the choice of $l$, while $C_{0}(E, q)$ does not. We now construct a particular Brauer class on $k\left(\mathbb{P}^{2}\right)$ restricting to $\beta_{X}$ on $k(S)$. This will play a crucial rôle in the proof of Theorem 1.

Proposition 4.3. Let $X$ be a smooth cubic fourfold containing a plane $P$ over a field $k$ of characteristic $\neq 2$. Assume that the associated quadric surface bundle has simple degeneration along a divisor $D \subset \mathbb{P}_{k}^{2}$ and let $S$ be the associated K3 surface of degree 2. Given a choice of homogeneous coordinates on $\mathbb{P}_{k}^{5}$, there exists a line $L \subset \mathbb{P}_{k}^{2}$ and a quaternion algebra $\alpha$ over $k\left(\mathbb{P}^{2}\right)$ whose restriction to $k(S)$ is isomorphic to the generic fiber of the Clifford invariant $\beta_{X}$, and such that $\alpha$ has ramification only at the generic points of $D$ and $L$.

Proof. For a choice of homogeneous coordinates on $\mathbb{P}_{k}^{5}$, let $L \subset \mathbb{P}_{k}^{2}$ be the line whose equation is $a_{00}$ from (2). The smoothness of $X$ implies that $a_{00}$ is nonzero. Then on $\mathbb{A}_{k}^{2}=\mathbb{P}_{k}^{2} \backslash L$, the choice of $a_{00}$ determines a trivialization $\psi:\left.\mathscr{O}(1)\right|_{\mathbb{A}_{k}^{2}} \rightarrow \mathscr{O}_{\mathbb{A}_{k}^{2}}$, with respect to which the quadratic form $q^{\prime}=\left.\psi \circ q\right|_{\mathbb{A}_{k}^{2}}:\left.\mathscr{E}\right|_{\mathbb{A}_{k}^{2}} \rightarrow \mathscr{O}_{\mathbb{A}_{k}^{2}}$ (given by the dehomogenization of equation (2) associated to $\psi$ ) represents 1 . Letting $V=$ $\mathbb{P}_{k}^{2} \backslash(D \cup L) \subset \mathbb{A}_{k}^{2}$, we have that $\left(\left.\mathscr{E}\right|_{V},\left.q^{\prime}\right|_{V}, \mathscr{O}_{V}\right)$ is a regular quadratic form of rank 
4 on $V$. In this case, we have the full Clifford algebra $\mathscr{C}=\mathscr{C}\left(\left.\mathscr{E}\right|_{V},\left.q^{\prime}\right|_{V}, \mathscr{O}_{V}\right)$ at our disposal, which is an Azumaya algebra of degree 4 on $V$.

Let us prove that $\left.\mathscr{C}\right|_{k\left(\mathbb{P}^{2}\right)}$ is Brauer equivalent to a symbol. Since $\left.q^{\prime}\right|_{k\left(\mathbb{P}^{2}\right)}$ represents 1 , we have a diagonalization

$$
\left.q^{\prime}\right|_{k\left(\mathbb{P}^{2}\right)}=<1, a, b, a b d>
$$

where $d \in k\left(\mathbb{P}^{2}\right)^{\times} / k\left(\mathbb{P}^{2}\right)^{\times 2}$ is the discriminant. Then in $\operatorname{Br}\left(k\left(\mathbb{P}^{2}\right)\right)$ we have

$$
\left[\left.\mathscr{C}\right|_{k\left(\mathbb{P}^{2}\right)}\right]=(a, b)+(a, a b d)+(b, a b d)+(-1,-d)=(-a b,-a d)
$$

by the formula [49, Ch. 2, Def. 12.7,Ch. 9, Rem. 2.12] relating the Clifford invariant to the 2nd Hasse-Witt invariant.

Letting $\alpha=(-a b,-a d) \in \operatorname{Br}\left(k\left(\mathbb{P}^{2}\right)\right)$, we see that $\alpha$ coincides with $\left[\left.\mathscr{C}\right|_{k\left(\mathbb{P}^{2}\right)}\right]$ in $\operatorname{Br}\left(k\left(\mathbb{P}^{2}\right)\right)$, hence is unramified at all codimension 1 points of $V$, i.e., $\alpha$ is ramified at most at the generic points of $D$ and $L$.

The even Clifford algebra is a similarity class invariant, hence we have an an isomorphism $\mathscr{C}_{0}\left(\left.\mathscr{E}\right|_{V},\left.q^{\prime}\right|_{V}, \mathscr{O}_{V}\right) \cong \mathscr{C}_{0}\left(\left.\mathscr{E}\right|_{V},\left.q\right|_{V},\left.\mathscr{O}(1)\right|_{V}\right)$ over $V$, hence over the inverse image of $V$ in $S$.

Finally, we have $\left.\mathscr{C}\right|_{k(S)} \cong M_{2}\left(\left.\mathscr{B}_{0}\right|_{k(S)}\right)$, hence $\alpha$ restricted to $k(S)$ is Brauer equivalent to the generic fiber of the Clifford invariant $\beta_{X} \in \operatorname{Br}(S)$.

\section{Proof of the MAIN RESUlT}

Let us recall the statement.

Theorem 5.1. Let $X \subset \mathbb{P}^{5}$ be a very general cubic fourfold containing a plane $P \subset \mathbb{P}^{5}$ over $\mathbb{C}$. Then $H_{\mathrm{nr}}^{3}(X / \mathbb{C}, \mathbb{Q} / \mathbb{Z}(2))$ is universally trivial.

Let $k$ be a field of characteristic $\neq 2$. Let $X \subset \mathbb{P}_{k}^{5}$ be a smooth cubic fourfold containing a plane over $k$. Let $\pi: \widetilde{X} \rightarrow \mathbb{P}_{k}^{2}$ be the associated quadric surface bundle. We assume that $\pi$ has simple degeneration along a smooth divisor $D \subset \mathbb{P}_{k}^{2}$, see Proposition 4.1. Denote by $Q$ the generic fiber of $\pi$; it is a smooth quadric surface over $k\left(\mathbb{P}^{2}\right)$. For any field extension $F / k$, we will need to refer to the following commutative diagram of Bloch-Ogus complexes

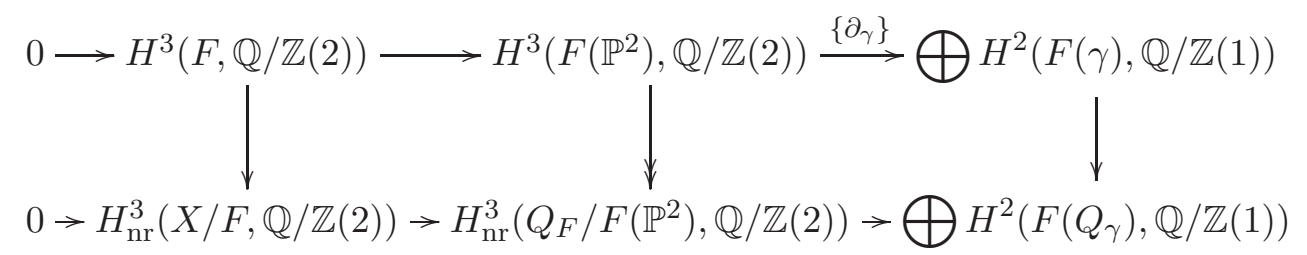

where the sums are taken over all points $\gamma$ of codimension 1 of $\mathbb{P}_{F}^{2}$. The top row is the exact sequence defining the unramified cohomology of $\mathbb{P}_{F}^{2}$ (which is constant, by Proposition 1.2) via the residue maps $\partial_{\gamma}$. The bottom row is the complex arising from taking residues on $F(Q)$ at points of codimension 1 of $\widetilde{X}$ whose image in $\mathbb{P}_{F}^{2}$ is of codimension 1 on $\mathbb{P}_{F}^{2}$ (recall that $H_{\mathrm{nr}}^{3}\left(Q_{F} / F\left(\mathbb{P}^{2}\right), \mathbb{Q} / \mathbb{Z}(2)\right)$ consists of classes over $F(Q)$ which have trivial residues at all rank 1 discrete valuations trivial on $\left.F\left(\mathbb{P}^{2}\right)\right)$. Here, $Q_{\gamma}$ denotes the generic fiber of the restricted quadric fibration $\left.\pi\right|_{C}:\left.\widetilde{X}\right|_{C} \rightarrow$ $C$, where $C \subset \mathbb{P}_{F}^{2}$ is a projective integral curve with generic point $\gamma$. Under the simple degeneration hypothesis, each $Q_{\gamma}$ is an integral quadric surface. The bottom complex is also exact. This is obvious except at the term $H_{\mathrm{nr}}^{3}\left(Q_{F} / F\left(\mathbb{P}^{2}\right), \mathbb{Q} / \mathbb{Z}(2)\right)$, 
and we shall not used the exactness at that point. The vertical maps in the diagram are the natural ones.

Let $\psi \in H_{\mathrm{nr}}^{3}\left(X_{F} / F, \mathbb{Q} / \mathbb{Z}(2)\right) \subset H_{\mathrm{nr}}^{3}\left(Q_{F} / F\left(\mathbb{P}^{2}\right), \mathbb{Q} / \mathbb{Z}(2)\right)$. By Theorem 3.3, this is the image of an element $\xi \in H^{3}\left(F\left(\mathbb{P}^{2}\right), \mathbb{Q} / \mathbb{Z}(2)\right)$. By the commutativity of the right-hand square, and since the bottom row of diagram (4) is a complex, for each $\gamma$ of codimension 1 in $\mathbb{P}_{F}^{2}$ we know that

$$
\partial_{\gamma}(\xi) \in \operatorname{ker}\left(H^{2}(F(\gamma), \mathbb{Q} / \mathbb{Z}(1)) \rightarrow H^{2}\left(F\left(Q_{\gamma}\right), \mathbb{Q} / \mathbb{Z}(1)\right)\right) .
$$

We recall that if $F$ is any field, then $H^{2}(F, \mathbb{Q} / \mathbb{Z}(1))$ is isomorphic to the prime-to- $p$ part of the Brauer group $\operatorname{Br}(F)$, where $p$ is the characteristic of $F$. With the notation of $\S 4$, where the line $L$ is defined by $a_{00}=0$, let $d=\Delta / a_{00}^{6}$. Then $\operatorname{div}(d)=D-6 L$, and the class of $d$ in $k\left(\mathbb{P}^{2}\right) / k\left(\mathbb{P}^{2}\right)^{\times 2}$ is the discriminant of the quadric $Q$.

Definition 5.2. Fix $L$ as above. Let $F / k$ be a field extension and $\xi$ a class in $H^{3}\left(F\left(\mathbb{P}^{2}\right), \mathbb{Q} / \mathbb{Z}(2)\right)$. We call an integral curve $C \subset \mathbb{P}_{F}^{2}$ with generic point $\gamma$ a bad curve (for $\xi$ ) if $C$ is different from $D_{F}$ and $L_{F}$ and if $\partial_{\gamma}(\xi) \neq 0$ in $H^{2}(F(\gamma), \mathbb{Q} / \mathbb{Z}(1)$ ).

There are finitely many bad curves for each given $\xi \in H^{3}\left(F\left(\mathbb{P}^{2}\right), \mathbb{Q} / \mathbb{Z}(2)\right)$. Let $\alpha \in H^{2}\left(k\left(\mathbb{P}^{2}\right), \mu_{2}\right)$ be the class of a quaternion algebra attached to $\pi: \widetilde{X} \rightarrow \mathbb{P}_{k}^{2}$ and the choice of a line $L$, as in Proposition 4.3. Theorem 3.1 and Proposition 3.2 imply that the following statements hold concerning bad curves:

a) The class $\left.d\right|_{\gamma} \in H^{1}\left(F(\gamma), \mu_{2}\right)$ is trivial.

b) The class $\left.\alpha\right|_{\gamma} \in H^{2}\left(F(\gamma), \mu_{2}\right)$ is nontrivial and coincides with $\left.c(Q)\right|_{\gamma} \in$ $\operatorname{Br}(F(\gamma))$.

c) The class $\partial_{\gamma}(\xi) \in H^{2}(F(\gamma), \mathbb{Q} / \mathbb{Z}(1))$ also coincides with $\left.c(Q)\right|_{\gamma} \in \operatorname{Br}(F(\gamma))$.

For curves $C$ split by the discriminant extension (e.g., for bad curves), we will construct special rational functions that are parameters along $C$ and are norms from the discriminant extension. This is where the very general hypothesis will be used.

Lemma 5.3. Let $k$ be a field of characteristic $\neq 2$. Let $r: S \rightarrow \mathbb{P}_{k}^{2}$ be a finite flat morphism of degree 2 branched over a smooth sextic curve D. Assume that $r^{*}: \operatorname{Pic}\left(\mathbb{P}_{k}^{2}\right) \rightarrow \operatorname{Pic}(S)$ is an isomorphism. Choose a line $L \subset \mathbb{P}_{k}^{2}$ and a function $d \in$ $k\left(\mathbb{P}^{2}\right)$ with divisor $D-6 L$ that satisfies $k(S)=k\left(\mathbb{P}^{2}\right)(\sqrt{d})$. If $C \subset \mathbb{P}_{k}^{2}$ is an integral curve (with generic point $\gamma$ ) different from $D$ and $L$ such that $\left.d\right|_{\gamma} \in H^{1}\left(k(\gamma), \mu_{2}\right)$ is trivial, then there exists a function $f_{\gamma} \in k\left(\mathbb{P}^{2}\right)$ whose divisor is $C-2 n L$ for some positive integer $n$, and which is a a norm from $k(S) / k\left(\mathbb{P}^{2}\right)$.

Proof. Under our hypothesis, $\operatorname{Pic}(S)=\mathrm{CH}^{1}(S)=\mathbb{Z} H$, where $H=r^{*} L$ by flat pullback. As a consequence, the action of $\operatorname{Aut}\left(S / \mathbb{P}^{2}\right)$ on $\operatorname{Pic}(S)=\mathrm{CH}^{1}(S)$ is trivial.

If the class of $d$ is a square in $k(\gamma)$, then $f^{-1} C$ splits as $C_{1} \cup C_{2}$, with $C_{1}$ and $C_{2}$ both having class $n H$ for some positive integer $n$. Hence $C_{1}-n H=\operatorname{div}\left(g_{\gamma}\right)$ for some $g_{\gamma} \in k(S)$. However, by proper push-forward, $r_{*}\left(C_{1}-n H\right)=C-2 n L$, hence $C-2 n L=\operatorname{div}\left(N_{k(S) / k\left(\mathbb{P}^{2}\right)}\left(g_{\gamma}\right)\right)$. Set $f_{\gamma}=N_{k(S) / k\left(\mathbb{P}^{2}\right)}\left(g_{\gamma}\right)$.

Remark 5.4. From the proof of Lemma 5.3, one sees that every bad curve $C$ has even degree. Using some further intersection theory, one can even prove that any such curve has degree 6 , though we shall not need this.

Now assume that $k=\mathbb{C}$. Let $\alpha \in \operatorname{Br}\left(\mathbb{C}\left(\mathbb{P}^{2}\right)\right)$ be the class of the full Clifford algebra $(\mathscr{E}, q, \mathscr{O}(1))$ over $\mathbb{P}^{2} \backslash(D \cup L)$, as in Proposition 4.3. Then $\alpha$ is unramified, except possibly at the generic points of $D$ and $L$. 
Now assume that $X$ is very general. Fix a field $F / \mathbb{C}$. For each bad curve $C$ over $F$, we will choose a parameter $f_{\gamma} \in F\left(\mathbb{P}^{2}\right)$ at the generic point $\gamma$ of $C$ as in Lemma 5.3, which we may apply since $r^{*}: \operatorname{Pic}\left(\mathbb{P}_{F}^{2}\right) \rightarrow \operatorname{Pic}\left(S_{F}\right)$ is an isomorphism by the rigidity of the Néron-Severi group. Denote by

$$
f=\prod f_{\gamma},
$$

where the product is taken over all bad curves.

Let us continue with the proof of Theorem 1. Recall that we have lifted $\psi \in$ $H_{\mathrm{nr}}^{3}\left(X_{F} / F, \mathbb{Q} / \mathbb{Z}(2)\right)$ to an element $\xi \in H^{3}\left(F\left(\mathbb{P}^{2}\right), \mathbb{Q} / \mathbb{Z}(2)\right)$. We now compute the residues of $\xi$ along codimension one points of $\mathbb{P}_{F}^{2}$, where we need only worry about the generic points of $D$, any bad curves $C$, and $L$.

Let us first consider the generic point $\eta$ of $D$. The hypothesis that $X$ is very general implies simple degeneration, hence that the $F(\eta)$-quadric $Q_{\eta}$ is a quadric cone over a smooth $F(\eta)$-conic. The conic is split by Tsen's theorem since it is the base change of a smooth $\mathbb{C}(\eta)$-conic. Hence Proposition 3.2, and the commutativity of the right hand square of diagram (4), implies that $\xi$ is unramified at $\eta$.

Now, we will compare $\xi$ with the class $\alpha \cup(f)$, whose ramification we control. Relying on Theorem 1.1, we are implicitly considering an inclusion $H^{3}\left(F\left(\mathbb{P}^{2}\right), \mu_{2}^{\otimes 2}\right) \subset$ $H^{3}\left(F\left(\mathbb{P}^{2}\right), \mathbb{Q} / \mathbb{Z}(2)\right)$. By construction, the function $f$ is a norm from the extension $F\left(\mathbb{P}^{2}\right)(\sqrt{d}) / F\left(\mathbb{P}^{2}\right)$, i.e., is of the form $f=g^{2}-d h^{2}$ for some $g, h \in F\left(\mathbb{P}^{2}\right)$. Also, $f$ has its zeros and poles only along the bad curves and $L$, hence in particular, $f$ is a unit at the generic point $\eta$ of $D$.

The extension $F\left(\mathbb{P}^{2}\right)(\sqrt{d}) / F\left(\mathbb{P}^{2}\right)$ is totally ramified at $\eta$. In particular, any unit in the local ring at $\eta$ which is a norm from $F\left(\mathbb{P}^{2}\right)(\sqrt{d})$ reduces to a square in the residue field $F(\eta)$. Thus $f$ lifts to a square in the completion $\left.\widehat{F\left(\mathbb{P}^{2}\right.}\right)_{\eta}$.

Now we consider the residues of $\alpha \cup(f) \in H^{3}\left(F\left(\mathbb{P}^{2}\right), \mu_{2}^{\otimes 2}\right)$ along codimension one points of $\mathbb{P}_{F}^{2}$. Both $\alpha \in H^{2}\left(F\left(\mathbb{P}^{2}\right), \mu_{2}\right)$ and $(f) \in H^{1}\left(F\left(\mathbb{P}^{2}\right), \mu_{2}\right)$ are unramified away from the generic points of $D, L$, and the bad curves $C$.

At the generic point $\eta$ of $D$, the function $f$ is a square in the completion $\widehat{F\left(\mathbb{P}^{2}\right)_{\eta}}$. Thus, the residue of $\alpha \cup(f)$ at $\eta$ is zero. At a bad curve, $\alpha$ is regular and the valuation of $f$ is one. Thus the residue at such a curve is $\left.\alpha\right|_{\gamma}=\left.c(Q)\right|_{\gamma}$.

Thus, we have that the difference $\xi-\alpha \cup(f) \in H^{3}\left(F\left(\mathbb{P}^{2}\right), \mathbb{Q} / \mathbb{Z}(2)\right)$ has trivial residues away from $L$, hence it comes from a constant class $\xi_{0}$ in the image of $H^{3}(F, \mathbb{Q} / \mathbb{Z}(2)) \rightarrow H^{3}\left(F\left(\mathbb{P}^{2}\right), \mathbb{Q} / \mathbb{Z}(2)\right)$.

Now we show that $\alpha \cup(f)$ vanishes when restricted to $H^{3}\left(F(Q), \mu_{2}^{\otimes 2}\right)$. In the notation of the proof of Proposition $4.3,\left.q\right|_{F\left(\mathbb{P}^{2}\right)}=<1, a, b, a b d>$ becomes isotropic over $F(Q)$ and $\alpha=(-a b,-a d)$. Since $f$ is a norm from $F\left(\mathbb{P}^{2}\right)(\sqrt{d})$, Lemma 3.5 implies that $(a, b, f)$ is trivial in $H^{3}\left(F(Q), \mu_{2}^{\otimes 2}\right)$. Further, since $f$ is a norm from $F\left(\mathbb{P}^{2}\right)(\sqrt{d})$, we have $(d, f)=0$.

But then

$$
\alpha \cup(f)=(a b, a d, f)=(a, b, f)+(a, a, f)+(a b, d, f)
$$

is trivial in $H^{3}\left(F(Q), \mu_{2}^{\otimes 2}\right)$ as well. Thus $\psi \in H_{\mathrm{nr}}^{3}\left(X_{F} / F, \mathbb{Q} / \mathbb{Z}(2)\right)$ is the image of the constant class $\xi_{0} \in H^{3}(F, \mathbb{Q} / \mathbb{Z}(2))$.

Remark 5.5. We can give a different argument, using Arason's result (see Theorem 3.4), for the vanishing of $\alpha \cup(f)$ in $H^{3}\left(F(Q), \mu_{2}^{\otimes 2}\right)$. As in the notation of the 
proof of Proposition 4.3, we write $q_{k\left(\mathbb{P}^{2}\right)}=<1, a, b, a b d>$ and $\alpha=(-a b,-a d)$. The 3-Pfister form associated to $\alpha \cup(f)$ decomposes as

$$
\ll-a b,-a d, f \gg=<1, a b, a d, b d\rangle \perp-f<1, a b, a d, b d\rangle
$$

Since $f$ is a norm from $F\left(\mathbb{P}^{2}\right)(\sqrt{d})$ we have that $d$ is a norm from $F\left(\mathbb{P}^{2}\right)(\sqrt{f})$. Thus $d$ is a similarity factor of the norm form of $F\left(\mathbb{P}^{2}\right)(\sqrt{f}) / F\left(\mathbb{P}^{2}\right)$, i.e., we have an isometry $<1,-f>\cong<d,-d f>$. Hence the 3-Pfister form

$$
\begin{aligned}
\ll-a b,-a d, f \gg & =<1, a b, a d, b d>\perp-f<1, a b, a d, b d> \\
& =<a b, a d, b d>\perp<1,-f>\perp-f<a b, b d, a d> \\
& =<a b, a d, b d>\perp<d,-d f>\perp-f<a b, b d, a d> \\
& =<d, a b, a d, b d>\perp-f<d, a b, b d, a d>
\end{aligned}
$$

contains the form $\langle d, a d, b d, a b\rangle=d<1, a, b, a b d\rangle$. Thus by Theorem 3.3, we have that $\alpha \cup(f)$ is trivial in $H_{\mathrm{nr}}^{3}\left(Q_{F} / F\left(\mathbb{P}^{2}\right), \mathbb{Q} / \mathbb{Z}(2)\right)$.

Corollary 5.6. Let $X \subset \mathbb{P}^{5}$ be a very general cubic fourfold containing a plane $P \subset \mathbb{P}^{5}$ over $\mathbb{C}$. Then $H_{\mathrm{nr}}^{3}\left(X / \mathbb{C}, \mu_{2}^{\otimes 2}\right)$ is universally trivial.

Proof. In the following commutative diagram

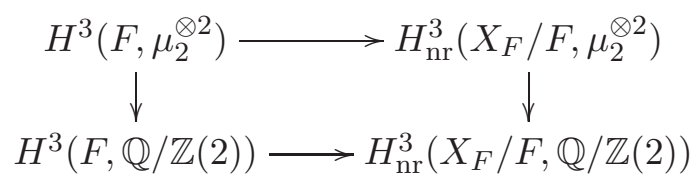

the horizontal maps are injective since we may specialize to an $F$-rational point. The vertical maps are injective by Theorem 1.1. By Theorem 5.1, any $\psi \in H_{\mathrm{nr}}^{3}\left(X_{F} / F, \mu_{2}^{\otimes 2}\right) \subset$ $H_{\mathrm{nr}}^{3}\left(X_{F} / F, \mathbb{Q} / \mathbb{Z}(2)\right)$ is the image of a constant class $\xi_{0} \in H^{3}(F, \mathbb{Q} / \mathbb{Z}(2))$, which by the diagram is 2-torsion, hence comes from an element in $H^{3}\left(F, \mu_{2}^{\otimes 2}\right)$. Since the right hand side vertical map is injective, the proof is complete.

\section{REFERENCES}

[1] J. Kr. Arason, Cohomologische Invarianten quadratischer Formen, J. Algebra 36 (1975), 448491. 11,12

[2] M. Artin, and D. Mumford, Some elementary examples of unirational varieties which are not rational, Proc. London Math. Soc. (3) 25 (1972), 75-95. 8

[3] A. Auel, M. Bernardara, and M. Bolognesi, Fibrations in complete intersections of quadrics, Clifford algebras, derived categories and rationality problems, preprint arXiv:1109.6938, 2013, Journal de Mathématiques Pures et Appliquées, to appear. 13

[4] A. Auel, M. Bernardara, M. Bolognesi, and A. Várilly-Alvarado, Cubic fourfolds containing a plane and a quintic del Pezzo surface, preprint arXiv:1205.0237. 1

[5] A. Auel, R. Parimala, and V. Suresh, Quadric surface bundles over surfaces, preprint arXiv:1207.4105. 14

[6] R. Barlow, Rational equivalence on zero cycles for some more surfaces with $p_{g}=0$, Invent. math. 79 (1985), 303-308. 7

[7] A. Beauville, Variétés de Prym et jacobiennes intermédiaires, Ann. Sci. École Norm. Sup. (4) 10, no. 3, 1977, 309-391. 13

[8] A. Beauville and R. Donagi, La variété des droites d'une hypersurface cubique de dimension 4, C.R. Acad. Sc. Paris, Série I 301 (1985), 703-706. 1

[9] S. Bloch, Lectures on Algebraic Cycles, Duke Univ. Math. Ser. IV, Durham, 1980. Second edition. New Mathematical Monographs 16. Cambridge University Press, Cambridge, 2010. 6

[10] S. Bloch, A. Kas, and D. Lieberman, Zero-cycles on surfaces with $p_{g}=0$, Compositio math. 33 (1976) 135-145. 7 
[11] S. Bloch and A. Ogus, Gersten's conjecture and the homology of schemes, Ann. Sci. École Norm. Sup. (4) 7 (1974), 181-201. 10

[12] S. Bloch and V. Srinivas, Remarks on correspondences and algebraic cycles, Amer. J. Math. 105 (1983), no. 5, 1235-1253. 6, 7

[13] F. Campana, Connexité rationnelle des variétés de Fano, Ann. Sci. École Norm. Sup. (4) 25 (1992), no. 5, 539-545. 7

[14] L. Campedelli, Sopra alcuni piani doppi notevoli con curve di diramazione d el decimo ordine, Atti Acad. Naz. Lincei 15 (1932), 536-542. 7

[15] G. Castelnuovo, Sulle superficie di genere zero, Memorie della Soc.It. delle Scienze (detta dei XL), ser. III, t. 10, (1896). 7

[16] C.H. Clemens and P. A. Griffiths, The intermediate Jacobian of the cubic threefold Ann. of Math. (2) 95 (1972), 281-356. 9

[17] J.-L. Colliot-Thélène, Birational invariants, purity and the Gersten conjecture, K-theory and algebraic geometry: connections with quadratic forms and division algebras (Santa Barbara, CA, 1992), Amer. Math. Soc., Proc. Sympos. Pure Math. 58 (1995), 1-64. 2, 3, 4

[18]_Formes quadratiques multiplicatives et variétés algébriques : deux compléments, Bulletin de la Société Mathématique de France 108 (1980), 213-227. 2

[19] Un théorème de finitude pour le groupe de Chow des zéro-cycles d'un groupe algébrique linéaire sur un corps p-adique, Invent. math. 159 (2005), no. 3, 589-606. 5, 8

[20] Quelques cas d'annulation du troisième groupe de cohomologie non ramifiée, Contempory Math. 571 (2012), 45-50. 10

[21] J.-L. Colliot-Thélène and D. Coray, L'équivalence rationnelle sur les points fermés des surfaces rationnelles fibrées en coniques, Compositio Math. 39 (1979), no. 3, 301-332. 4

[22] J.-L. Colliot-Thélène and W. Raskind, $\mathscr{K}_{2}$-cohomology and the second Chow group, Math. Ann. 270 (1985), no. 2, 165-199. 7

[23] J.-L. Colliot-Thélène and J.-J. Sansuc, Cohomologie des groupes de type multiplicatif sur les schémas réguliers, C. R. Acad. Sci. Paris Sér. A-B, no. 6, 287 (1978), A449-A452. 10

[24] J.-L. Colliot-Thélène and A.N. Skorobogatov, Groupe de Chow des zéro-cycles sur les fibrés en quadriques, K-Theory 7 (1993), no. 5, 477-500. 11

[25] J.-L. Colliot-Thélène and C. Voisin, Cohomologie non ramifiée et conjecture de Hodge entière, Duke Math. J. 161 (2012), no. 5, 735-801. 10

[26] F. Enriques, Introduzione alla geometria sopra le superficie algebriche, Memorie della Societa' Italiana delle Scienze (detta "dei XL"), s.3 , to. X, (1896), 1-81. 7

[27] _ Memorie scelte di geometria, vol. I, Zanichelli, Bologna, 1956. 7

[28] H. Esnault, Varieties over a finite field with trivial Chow group of 0-cycles have a rational point, Invent. math. 151 (2003), no. 1, 187-191. 7

[29] G. Fano, Sulle forme cubiche dello spazio a cinque dimensioni contenenti rigate rationali di quarto ordine, Comment. Math. Helv. 15 (1943), 71-80. 1

[30] W. Fulton, Intersection theory, Ergebnisse der Mathematik und ihrer Grenzgebiete (3) vol. 2, Springer-Verlag, Berlin, 1984. 4, 5

[31] L. Godeaux, Les involutions cycliques appartenant à une surface algébrique, Actual. Sci. Ind. 270, Hermann, Paris, 1935. 7

[32] J. Harris, B. Mazur, and R. Pandharipande, Hypersurfaces of low degree, Duke Math. J. 95 (1998), no. 1, 125-160. 9

[33] B. Hassett, Special cubic fourfolds, Compos. Math. 120 (2000), no. 1, 1-23. 1, 13

[34] _ Some rational cubic fourfolds, J. Algebraic Geometry 8 (1999), no. 1, 103-114. 1, 13

[35] B. Hassett and Y. Tschinkel, Rational curves on holomorphic symplectic fourfolds, Geom. Funct. Anal. 11 (2001), no. 6, 1201-1228. 9

[36] B. Kahn, Bruno, M. Rost, and R. Sujatha, Unramified cohomology of quadrics. I, Amer. J. Math. 120 (1998), no. 4, 841-891. 5, 11

[37] N.A. Karpenko and A.S. Merkurjev, On standard norm varieties, Ann. Sc. Éc. Norm. Sup. 46 (2013), 175-214. 5

[38] M.-A. Knus, Quadratic and hermitian forms over rings, Springer-Verlag, Berlin, 1991. 13

[39] M.-A. Knus, A. Merkurjev, M. Rost, and J.-P. Tignol, The Book of Involutions, Colloquium Publications, vol. 44, AMS, 1998. 14 
[40] M.-A. Knus, R. Parimala, and R. Sridharan, On rank 4 quadratic spaces with given Arf and Witt invariants, Math. Ann. 274 (1986), no. 2, 181-198. 1

[41] J. Kollár, Y. Miyaoka, and S. Mori, Rational connectedness and boundedness of Fano manifolds, J. Differential Geom. 36 (1992), no. 3, 765-779. 7

[42] A. Kuznetsov, Derived categories of quadric fibrations and intersections of quadrics, Adv. Math. 218 (2008), no. 5, 1340-1369. 13

[43] A.S. Merkurjev, Unramified elements in cycle modules, J. Lond. Math. Soc. (2) 78 (2008), no. $1,51-64.2,5$

[44] J.P. Murre, On the Hodge conjecture for unirational fourfolds, Nederl. Akad. Wetensch. Proc. Ser. A 80 (Indag. Math. 39) (1977), no. 3, 230-232. 2, 10

[45] B. Poonen and J.F. Voloch (with appendices by J.-L. Colliot-Thélène and N.M. Katz), Random diophantine equations, Arithmetic of Higher-Dimensional Algebraic Varieties, (Bjorn Poonen and Yuri Tschinkel, eds.), pp. 175-184, Progress in Mathematics, Vol. 226, 2004, Birkhäuser Boston, Cambridge, MA. 2, 10

[46] M. Reid, Undergraduate algebraic geometry, London Mathematical Society Student Texts, vol. 12, Cambridge University Press, Cambridge, 1988. 9

[47] T. Reye, Die Geometrie der Lage, Vorträge, Baumgärtner, Leipzig, 1882. 7

[48] M. Rost, Chow groups with coefficients, Doc. Math.1 (1996), no. 16, 319-393. 4

[49] W. Scharlau, Quadratic and Hermitian forms, Grundlehren der Mathematischen Wissenschaften 270, Springer-Verlag, Berlin, 1985. 15

[50] R. Swan, Zero cycles on quadric hypersurfaces, Proc. Amer. Math. Soc. 107 (1989), no. 1, 43-46. 5

[51] S.L. Tregub, Three constructions of rationality of a cubic fourfold, Vestnik Moskov. Univ. Ser. I Mat. Mekh. (1984), no. 3, 8-14. Translation in Moscow Univ. Math. Bull. 39 (1984), no. 3, 8-16. 1

[52] _ Two remarks on four-dimensional cubics, Uspekhi Mat. Nauk 48 (1993), no. 2(290), 201-202. Translation in Russian Math. Surveys 48 (1993), no. 2, 206-208. 1

[53] C. Voisin, Bloch's conjecture for Catanese and Barlow surfaces, preprint arXiv:1210.3935, 2013, Journal of Differential Geometry (volume dedicated to the memory of Friedrich Hirzebruch), to appear. 7

[54] — Théorème de Torelli pour les cubiques de $\mathbb{P}^{5}$, Invent. math. 86 (1986), no. 3, 577-601. 13

[55] Hodge theory and complex algebraic geometry. I, II. Translated from the French by Leila Schneps. Cambridge Studies in Advanced Mathematics 76, 77, Cambridge University Press, Cambridge, 2007. 7

[56] Some aspects of the Hodge conjecture, Jpn. J. Math. 2 (2007), no. 2, 261-296. 2, 10

[57] E. Witt, Über ein Gegenbeispiel zum Normensatz, Math. Z. 39 (1935), no. 1, 462-467. 11

[58] S. Zucker, The Hodge conjecture for cubic fourfolds, Compositio Math. 34 (1977), no. 2, 199209. 2,10

Asher Auel, Department of Mathematics, Yale University, 10 Hillhouse Avenue, New Haven, CT 06511, USA E-mail address: asher.auel@yale.edu

R. Parimala, Department of Mathematics \& CS, Emory University, 400 Dowman Drive, Atlanta, GA 30322, USA E-mail address: parimala@mathcs.emory.edu

Jean-Louis Colliot-Thélène, CNRS, Université Paris-Sud, Mathématiques, BÂtiment 425, 91405 Orsay Cedex, France

E-mail address: jlct@math.u-psud.fr 